\title{
Crescimento de leguminosas utilizadas na adubação verde em diferentes níveis de sais na água de irrigação
}

\author{
Roseclênia A. Santos ${ }^{1}$, Paulo T. Carneiro ${ }^{2}$, Valdevan R. Santos ${ }^{3}$, \\ Leonardo C. Costa ${ }^{4}$, Cícero G. dos Santos ${ }^{5}$ \& Antônio L. dos Santos Neto ${ }^{6}$ \\ ${ }^{1}$ ESALQ/USP, Piracicaba, SP. E-mail: roseclenia.alves@usp.br \\ ${ }^{2}$ UFAL, Campus Arapiraca, Arapiraca, AL. E-mail: ptcarneiro@yahoo.com.br \\ ${ }^{3}$ UFAL, Campus Arapiraca, Arapiraca, AL. E-mail: valdevan@yahoo.com.br (Autor correspondente) \\ ${ }^{4}$ UNESP, Campus Jaboticabal, SP. E-mail: leonardocorreia08@gmail.com \\ ${ }^{5}$ UFAL, Campus Arapiraca, Arapiraca, AL. E-mail: gomes@arapiraca.ufal.br \\ ${ }^{6}$ UFAL, Campus Arapiraca, Arapiraca, AL. E-mail: santosneto@gmail.com
}

\section{Palavras-chave:}

salinidade

plantas de cobertura

biometria

\begin{abstract}
R E S U M O
Objetivou-se, com este trabalho, avaliar a influência da salinidade no crescimento de espécies de leguminosas comumente utilizadas como adubação verde. Conduziu-se um experimento em ambiente protegido, no ano de 2010, em blocos ao acaso, no esquema fatorial 5 x 2. Os tratamentos foram cinco níveis de sais $(0,8,1,6,2,4,3,2 \mathrm{e}$ $4,0 \mathrm{dS} \mathrm{m}^{-1}$ a $25^{\circ} \mathrm{C}$ ) e duas leguminosas: Crotalaria juncea e Crotalaria spectabilis. A água de irrigação foi preparada com a adição de $\mathrm{NaCl}$ e as irrigações foram feitas manualmente, em dias alternados, com base no consumo de água das plantas na irrigação anterior. Avaliaram-se os componentes morfofisiológicos - altura de plantas, diâmetro do caule e número de folhas. Plantas de Crotalaria juncea não foram afetadas pelos níveis de sais utilizados neste trabalho. Observou-se efeito depreciativo dos níveis de sais aos 40 dias, para altura de plantas, diâmetro do caule e número de folhas da Crotalaria spectabilis, com resposta linear para altura de planta e quadrática para diâmetro do caule e número de folhas.
\end{abstract}

Key words:

salinity

cover crops

biometry

\section{Growth of leguminous plants used as green manure under different salt levels in irrigation water}

\begin{abstract}
A B S T R A C T
This study had the objective to evaluate the influence of salinity on the growth of legumenous species commonly used as green manure. An experiment was conducted in a greenhouse in the year of 2010 set up in $5 \times 2$ factorial in randomized blocks. Treatments were composed of five levels of salt $\left(0.8,1.6,2,4,3.2\right.$ and $4.0 \mathrm{dS} \mathrm{m}^{-1}$ at $\left.25^{\circ} \mathrm{C}\right)$ in irrigation water and two legumenous species: Crotalaria juncea and Crotalaria spectabilis. Sodium chloride was added in the irrigation water and plants were irrigated manually on alternate days, based on water consumption of the previous day. The morpho-physiological parameters - plant height, stem diameter and number of leaves, were evaluated. Absolute growth rates and relative growth rates were adjusted to the regression models. Plants of Crotalaria juncea were not affected with salt levels used in this study. Depressive effect of salt levels was observed at 40 days for plant height, stem diameter and number of leaves of Crotalaria spectabilis, with linear response for plant height and quadratic for stem diameter and number of leaves.
\end{abstract}

\section{INTRODUÇÃo}

A adubação verde é uma prática que tem contribuído para a sustentabilidade da agricultura, principalmente por sua melhoria nas características química, física e biológicas do solo (Alcântara et al., 2000; Cunha et al., 2011) resultando em melhores rendimentos para as culturas em sucessão ou rotação.

A eficiência das plantas utilizadas como adubação verde em produzir resíduos, favorece o aumento no teor de matéria orgânica do solo, aumenta a disponibilidade de nutrientes (Fontanétti et al., 2006), melhora a habilidade de reciclagem e a mobilização de nutrientes lixiviados de camadas mais profundas e ainda eleva a capacidade de trocas de cátions efetiva (Alcântara et al., 2000). Essas características proporcionam um ambiente mais favorável para o desenvolvimento das plantas.

Espécies da família das leguminosas (Fabaceae) são as plantas mais utilizadas na adubação verde em função de sua capacidade em realizar simbiose com bactérias, especialmente do gênero Rhizobium, as quais conseguem fixar nitrogênio do ar atmosférico e incorporá-lo ao solo através da biomassa das plantas, sendo um suprimento efetivo de $\mathrm{N}$ para as culturas subsequentes, sobretudo as hortaliças (Vargas et al., 2011). O uso de adubos verdes como adubação complementar às culturas, é uma alternativa importante em razão de reduzir as limitações 
dos nutrientes, sobretudo nitrogênio e os custos com a aquisição de fertilizantes inorgânicos.

Dentre as espécies de leguminosas (Fabaceae) utilizadas na adubação verde se destacam a Crotalaria juncea L. e Crotalaria spectabilis Roth, por serem plantas de rápido crescimento vegetativo, eficientes na produção de biomassa e extração de nutrientes, além de adaptadas às condições de baixa fertilidade do solo (Alcântara et al., 2000; Fontanétti et al., 2006; Vargas et al., 2011), com ciclo de cultivo relativamente curto (Carneiro et al., 2008). Porém os efeitos promovidos por essas plantas podem ser influenciados por fatores abióticos (Alcântara et al., 2000). Desta forma, para o uso de espécies como adubos verdes, especialmente em regiões áridas e semiáridas, deve-se levar em consideração a salinidade do solo, visto que este é um dos fatores ambientais mais limitantes na produtividade das culturas (Zhu et al., 2008 ).

Segundo Zhu (2001) aproximadamente $20 \%$ das áreas cultivadas em todo o mundo e quase metade das áreas irrigadas, estão afetadas por sais, cujos solos salinos estão amplamente distribuídos nessas áreas secas. As regiões de clima seco necessitam de intensa irrigação para a produção das culturas; em geral há, nessas regiões, a ocorrência de fontes de água salina o que, aliado à alta evapotranspiração, provoca o acúmulo de sais no solo (Mahajan \& Tuteja, 2005; Kudo et al., 2010).

Altas concentrações de sais na zona radicular provocam alterações nas respostas fisiológicas das plantas (Parida \& Das, 2005) como desequilíbrio no balanço osmótico, desorganização das membranas, inibição na divisão e expansão celular (Mahajan \& Tuteja, 2005) características que contribuem para a diminuição do crescimento das plantas, evento passível de se manifestar em reduções em altura de planta, no número de folhas e no diâmetro do caule (Zhu et al., 2008; Silva et al., 2009; Oliveira et al., 2009) na área foliar e na produção de fitomassa (Oliveira et al., 2009; Neves et al., 2009; Santos et al., 2010).

Como o crescimento é avaliado por meio de variações de tamanho de algum aspecto da planta, geralmente morfológico, ele passa a ser o fator fisiológico de maior importância para a análise de crescimento, a qual permite selecionar cultivares ou espécies que apresentem características funcionais mais apropriadas ao objetivo do estudo (Benincasa, 2003). A taxa de crescimento, o número de folhas, a altura de planta e o diâmetro do caule, são bons parâmetros para avaliar os efeitos do excesso de sais na planta e na habilidade da planta quanto a sua tolerância à salinidade, visto que os processos de crescimento são particularmente sensíveis ao estresse salino (Parida \& Das, 2005).

A tolerância à salinidade é variável entre espécies (Kudo et al., 2010) e até mesmo dentro da espécie (Mendonça et al., 2007; Zhu et al., 2008) e depende de diversos fatores, como estádio fenológico (Bustingorri \& Lavado, 2011), intensidade e duração do estresse salino (Neves et al., 2009).

As espécies de leguminosas Crotalaria juncea e Crotalaria spectabilis devem apresentar respostas diferenciadas à salinidade; entretanto, em virtude da importância e da ampla utilização dessas plantas como adubos verdes, pesquisas que avaliem a influência da salinidade nos componentes de crescimento dessas espécies são fundamentais de vez que existem poucos estudos neste sentido ou são praticamente inexistentes.

Desta forma objetivou-se, com este trabalho, avaliar a influência de diferentes níveis de sais na água de irrigação e nos componentes de crescimento das espécies de leguminosas Crotalaria juncea e Crotalaria spectabilis.

\section{Material e Métodos}

O experimento foi desenvolvido entre os meses de novembro e dezembro de 2010, em ambiente protegido do Campus de Arapiraca da Universidade Federal de Alagoas (UFAL) município de Arapiraca, AL, localizado nas coordenadas geográficas de $09^{\circ} 42^{\prime} 02^{\prime \prime}$ de latitude Sul e $36^{\circ} 41^{\prime} 12^{\prime \prime}$ de longitude Oeste e altitude média de $325 \mathrm{~m}$. É uma região de transição entre a Zona da Mata e o Sertão Alagoano e, de acordo com o critério de classificação de Köppen, o clima da região é do tipo 'As', tropical, apresentando duas estações climáticas bem definidas, verão quente e seco, com chuvas eventuais (setembro a março) e inverno úmido e chuvoso (abril a agosto). A precipitação média anual varia entre 700 e 1100 $\mathrm{mm}$ e temperaturas médias anuais de $26,5^{\circ} \mathrm{C}$.

$\mathrm{O}$ experimento foi instalado em vasos de PVC, com dimensões de $0,40 \mathrm{~m}$ de altura e $0,20 \mathrm{~m}$ de diâmetro, perfurados na base para monitorar a água de drenagem. A massa de solo utilizada para encher os vasos foi oriunda de um Argissolo Vermelho distrófico (EMBRAPA, 2006) não salino e não sódico.

Os tratamentos consistiram de cinco níveis de salinidade (S) expressos em termos de condutividade elétrica da água (CEa) de irrigação: S1 - 0,8; S2 - 1,6; S3 - 2,4; S4 - 3,2 e S5 - 4,0 dS m ${ }^{-1}(25$ $\left.{ }^{\circ} \mathrm{C}\right)$ testadas em duas espécies de leguminosas: Crotalaria juncea L. e Crotalaria spectabilis Roth. Utilizou-se o delineamento experimental de blocos casualizados em esquema fatorial $5 \mathrm{x}$ 2, com seis repetições. Para cada espécie foram semeadas cinco sementes por vaso, com desbaste realizado aos sete dias após a semeadura, deixando-se apenas duas plantas por vaso.

A água de irrigação foi preparada pela adição de $\mathrm{NaCl}$, na qual se multiplicou o valor desejado da condutividade elétrica $\left(\mathrm{dS} \mathrm{m}{ }^{-1}\right.$ ) por $640 \mathrm{mg} \mathrm{L}^{-1}$, conforme Richards (1954). As irrigações foram feitas manualmente, em dias alternados e no final da tarde, com base no consumo de água das plantas na irrigação anterior. $\mathrm{O}$ volume estimado foi dividido pelo fator 0,8 , com o intuito de restabelecer a umidade do solo à capacidade de campo e obter uma fração de lixiviação (FL) de aproximadamente $20 \%$, com a utilização da seguinte equação:

$$
\mathrm{VI}=\frac{\mathrm{VA}-\mathrm{VD}}{1-\mathrm{FL}}
$$

em que:

VI - volume de água a ser aplicado na irrigação, $\mathrm{mL}$

VA - volume de água aplicado na irrigação anterior, $\mathrm{mL}$

VD - volume de água drenado na irrigação anterior, $\mathrm{mL}$

A avaliação do crescimento das plantas foi realizada até os quarenta dias após a aplicação dos tratamentos salinos (DAAT) 
com início dez dias após a semeadura das leguminosas. A altura da planta foi medida do colo até o meristema apical, por meio de uma trena graduada ( $\mathrm{cm}$ e $\mathrm{mm}$ ); estabeleceu-se, para a determinação do diâmetro do caule, uma altura padrão de $2 \mathrm{~cm}$ acima do nível do solo em que os dados foram obtidos por meio de paquímetro e, por fim, foi contado o número de folhas das plantas.

A partir dos valores médios obtidos para altura de planta e diâmetro do caule, foram calculadas suas respectivas taxas de crescimento absoluto (TCA) e taxas de crescimento relativo (TCR) conforme metodologia preconizada por Benincasa (2003), por meio das seguintes equações:

$$
\begin{gathered}
\mathrm{TCA} \frac{\mathrm{V} 2 \mathrm{~V} 1}{\mathrm{~T} 2 \mathrm{~T} 1} \\
\mathrm{TCR}=\frac{\ln \mathrm{V} 2-\ln \mathrm{V} 1}{\mathrm{~T} 2-\mathrm{T} 1}
\end{gathered}
$$

em que:

TCA - taxa de crescimento absoluto, $\mathrm{cm} \mathrm{d}^{-1}$ para altura e $\mathrm{mm} \mathrm{d}^{-1}$ para diâmetro

TCR - taxa de crescimento relativo, $\mathrm{cm} \mathrm{cm}^{-1} \mathrm{~d}^{-1}$ para altura e $\mathrm{mm} \mathrm{mm}^{-1} \mathrm{~d}^{-1}$ para diâmetro

ln - logaritimo natural

$\mathrm{V}_{1}$ e $\mathrm{V}_{2}$ - altura da planta ou diâmetro do caule, de duas amostras sucessivas em intervalos de tempo

$\mathrm{T}_{1}$ e $\mathrm{T}_{2}$ - intervalo de tempo, 20 dias

Os valores médios de número de folhas, altura de plantas e diâmetro do caule foram analisados em cada época de avaliação em função da condutividade elétrica da água de irrigação. Os dados obtidos foram submetidos à análise de variância pelo teste $\mathrm{F}(\mathrm{p}<0,05)$ e os efeitos significativos comparados pela análise de regressão por meio do software SISVAR ${ }^{\circledast}$ (Ferreira, 2011).

\section{Resultados e Discussão}

O resumo da análise de variância para o crescimento da Crotalaria juncea em altura de planta, diâmetro do caule e número de folhas, aos 20 e 40 dias após a aplicação dos tratamentos salinos, é apresentado na Tabela 1. Verificouse que os diferentes níveis de salinidade não afetaram significativamente nenhuma dessas variáveis; entrentanto, Nunes et al. (2009) observaram efeito prejudicial de fontes e concentrações salinas na germinação de sementes e no crescimento inicial de plântulas de Crotalaria juncea. Essas informações indicam que a utilização de água salina imediatamente após a semeadura desta espécie, poderia favorecer o aparecimento de sintomas de toxidez no período de avaliação do experimento em virtude dos níveis de sais da água de irrigação interagirem já a partir da semeadura.

A ausência de significância da condutividade elétrica da água (CEa) de irrigação sobre as variáveis de crescimento da Crotalaria juncea avaliadas neste trabalho, pode estar associada aos mecanismos de tolerância de algumas plantas ao estresse salino, dentre eles seletividade na acumulação, exclusão ou compartimentalização de íons específicos nas células, controle na absorção desses íons pelas raízes e transporte dentro das folhas ou, ainda, indução de enzimas antioxidantes (Parida \& Das, 2005).

Os resultados obtidos neste trabalho são semelhantes aos de Amorim et al. (2002) que também não observaram efeito negativo da salinidade sobre a altura do pseudocaule, número de folhas e diâmetro do colmo de plantas de alho até os 30 dias após plantio. Da mesma forma, os autores também têm relatado ausência de influência dos sais em variáveis de crecimento de alface, cultivar Verônica (Santos et al., 2010) e em Eucalyptus tereticornis (Mendonça et al., 2007).

Verifica-se, por outro lado, que aos 40 dias após a aplicação dos tratamentos a altura de planta, o diâmetro caulinar e o

Tabela 1. Resumo da análise de variância para altura de planta (AP), diâmetro de caule (DC) e número de folhas (NF) das

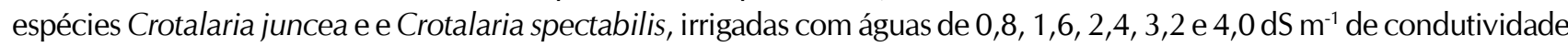

\begin{tabular}{|c|c|c|c|c|c|c|c|c|c|c|}
\hline \multirow{3}{*}{ Fonte de variação } & \multirow{3}{*}{ GL } & \multicolumn{3}{|c|}{ AP } & \multicolumn{3}{|c|}{ DC } & \multicolumn{3}{|c|}{ NF } \\
\hline & & \multicolumn{9}{|c|}{ Quadrados médios (DAAT) } \\
\hline & & 0 & 20 & 40 & 0 & 20 & 40 & 0 & 20 & 40 \\
\hline & & \multicolumn{9}{|c|}{ Crotalaria juncea } \\
\hline Salinidade & 4 & $13,15^{\mathrm{NS}}$ & 133,33 NS & 59,62 NS & 0,28 NS & 0,80 NS & $3,15^{\mathrm{NS}}$ & 2,72 NS & 99,08 NS & $1248,77^{\mathrm{NS}}$ \\
\hline Regressão linear & 1 & 18,33 NS & 289,52 NS & 60,40 NS & 0,86 NS & 2,56 ** & $7,35^{*}$ & 1,07 Ns & 74,82 NS & 3471,68 NS \\
\hline Regressão quadrática & 1 & 2,88 NS & 54,72 NS & 165,20 NS & 0,04 NS & 0,08 NS & 0,43 NS & 2,33 NS & 4,30 NS & 206,17 NS \\
\hline Desvio regressão & 2 & 15,69 NS & 94,54 NS & $6,45^{\mathrm{NS}}$ & $0,10^{\mathrm{NS}}$ & 0,27 NS & 2,42 NS & 3,73 NS & 158,61 NS & 658,61 NS \\
\hline Bloco & 5 & 15,40 NS & $224,47^{N S}$ & $132,13^{\mathrm{NS}}$ & 0,39 NS & $0,38^{N S}$ & 0,38 NS & $8,21^{\mathrm{NS}}$ & $83,76^{\mathrm{NS}}$ & 779,54 NS \\
\hline Resíduo & 20 & 7,76 & 139,04 & 151,41 & 0,40 & 0,33 & 1,45 & 5,20 & 121,84 & 1192,42 \\
\hline \multirow[t]{2}{*}{ CV (\%) } & & 15,63 & 17,00 & 10,85 & 26,07 & 11,09 & 14,05 & 16,36 & 20,44 & 22,22 \\
\hline & & \multicolumn{9}{|c|}{ Crotalaria spectabilis } \\
\hline Salinidade & 4 & 0,95 NS & 4,30 NS & $181,29^{* \star}$ & $0,25 \mathrm{NS}$ & $0,18^{\mathrm{NS}}$ & $7,49^{* *}$ & $0,47^{\mathrm{NS}}$ & $15,76^{\mathrm{NS}}$ & 3846,12 ** \\
\hline Regressão linear & 1 & 0,86 NS & 5,54 NS & 638,96 ** & 0,32 NS & 0,02 NS & $11,95^{* *}$ & 0,07 NS & 1,35 NS & $12183,75^{\text {** }}$ \\
\hline Regressão quadrática & 1 & 0,01 NS & 0,00 NS & 46,18 NS & 0,03 NS & 0,16 NS & $13,55^{\text {** }}$ & 0,76 NS & 6,86 NS & 2336,30 ** \\
\hline Desvio regressão & 2 & 1,46 NS & 5,83 NS & 20,01 NS & 0,33 NS & 0,27 NS & 2,23 * & 0,52 NS & $27,41^{\mathrm{NS}}$ & 432,30 * \\
\hline Bloco & 5 & $0,08^{N S}$ & $10,45^{N S}$ & 10,72 NS & $0,11^{\mathrm{NS}}$ & 0,22 NS & 0,70 NS & $1,58^{*}$ & $33,57^{\text {NS }}$ & 26,29 NS \\
\hline Resíduo & 20 & 0,73 & 4,52 & 13,49 & 0,10 & 0,10 & 0,49 & 0,45 & 15,32 & 50,88 \\
\hline CV (\%) & & 12,30 & 13,27 & 8,46 & 16,12 & 7,99 & 8,53 & 13,64 & 13,89 & 6,34 \\
\hline
\end{tabular}
elétrica, aos 0, 20 e 40 dias após aplicação dos tratamentos (DAAT)

${ }^{*} \mathrm{e}^{* *}$ significativo a 0,05 e 0,01 de probabilidade, respectivamente; ${ }^{\text {Ns }}$ não significativo 
número de folhas da Crotalaria spectabilis, foram afetados significativamente a nível de $\mathrm{p}<0,01$ (Tabela 1 ) com resposta linear para altura de planta; para o diâmetro do caule e para o número de folhas, constatou-se melhor ajustamento ao modelo quadrático.

O efeito depreciativo da salinidade sobre a altura de planta da Crotalaria spectabilis foi de $26 \%$ no maior nível $\left(4,0 \mathrm{dS} \mathrm{m}^{-1}\right)$ em relação ao menor $\left(0,8 \mathrm{dS} \mathrm{m}^{-1}\right)$. Houve redução de $4 \mathrm{~cm}$ em altura para cada unidade de condutividade elétrica da água de irrigação (Figura 1A).

Efeito da salinidade sobre a altura de planta de outras culturas, também foi observado. Oliveira et al. (2009) verificaram, considerando a diferença entre os extremos dos níveis salinos (0,5 e 5,0 dS $\left.\mathrm{m}^{-1}\right)$ uma redução de $16,4 \%$ na cultura do milho. Reduções na altura de plantas de mamoneira foram observadas por Silva et al. (2008) quando submetidas ao estresse salino. Um dos efeitos mais comuns da salinidade nas plantas é a diminuição no crescimento visto que o excesso de sais no ambiente radicular provoca menor disponibilidade e absorção de água pelas raízes aumentando, consequentemente, a pressão osmótica que provoca inibição na expansão e divisão celular (Mahajan \& Tuteja, 2005) comprometendo a absorção de nutrientes.

Observou-se, em relação ao diâmetro do caule, estímulo positivo da salinidade até cerca de $2,0 \mathrm{dS} \mathrm{m}^{-1}$, a partir do qual houve efeito depreciativo sendo mais acentuado à medida em que se aumentou a concentração de sais na água de irrigação (Figura 1B). Nery et al. (2009) registraram redução no diâmetro do caule das plantas de amendoim em $7 \%$ por aumento unitário de condutividade elétrica da água de irrigação. Redução linear no diâmetro caulinar da mamoneira foi verificada por Silva et al. (2008) até o nível de $6,7 \mathrm{dS} \mathrm{m}^{-1}$ aos 100 dias após a semeadura.

Para o número de folhas observou-se que, praticamente, não houve interferência da salinidade até o nível de 1,6 dS m-1 (Figura $1 \mathrm{C}$ ) porém decresceu em $44 \%$ na maior concentração de sais $\left(4,0 \mathrm{dS} \mathrm{m}^{-1}\right)$. Esses resultados indicam que as folhas são órgãos bastante afetados com o aumento da concentração de sais na água de irrigação. Oliveira et al. (2009) observaram, estudando o efeito dos sais em milho, redução de 33\% no número de folhas no nível de $3,5 \mathrm{dS} \mathrm{m}^{-1}$, em comparação ao menor nível de salinidade $\left(0,5 \mathrm{dS} \mathrm{m}^{-1}\right)$. Redução no número de folhas também foi observada por Silva et al. (2009) em feijão sempre que o nível salino da água de irrigação aumentou.

É provável que a redução no número de folhas em condições de salinidade seja um mecanismo de adaptação ao estresse, diminuindo a superfície transpirante. Esta redução pode ser devida à incapacidade da planta em produzir folhas novas mais rápido do que a senescência, além da morte das folhas mais velhas por necrose de seus tecidos, decorrente de efeitos tóxicos provocados por íons específicos provenientes do excesso de sais (Zhu, 2001; Mahajan \& Tuteja, 2005; Silva et al., 2009; Kudo et al., 2010).

Não se observou efeito dos níveis de sais da água de irrigação nas taxas de crescimento absoluto e relativo, nas características de crescimento altura de planta e diâmetro do caule, para a
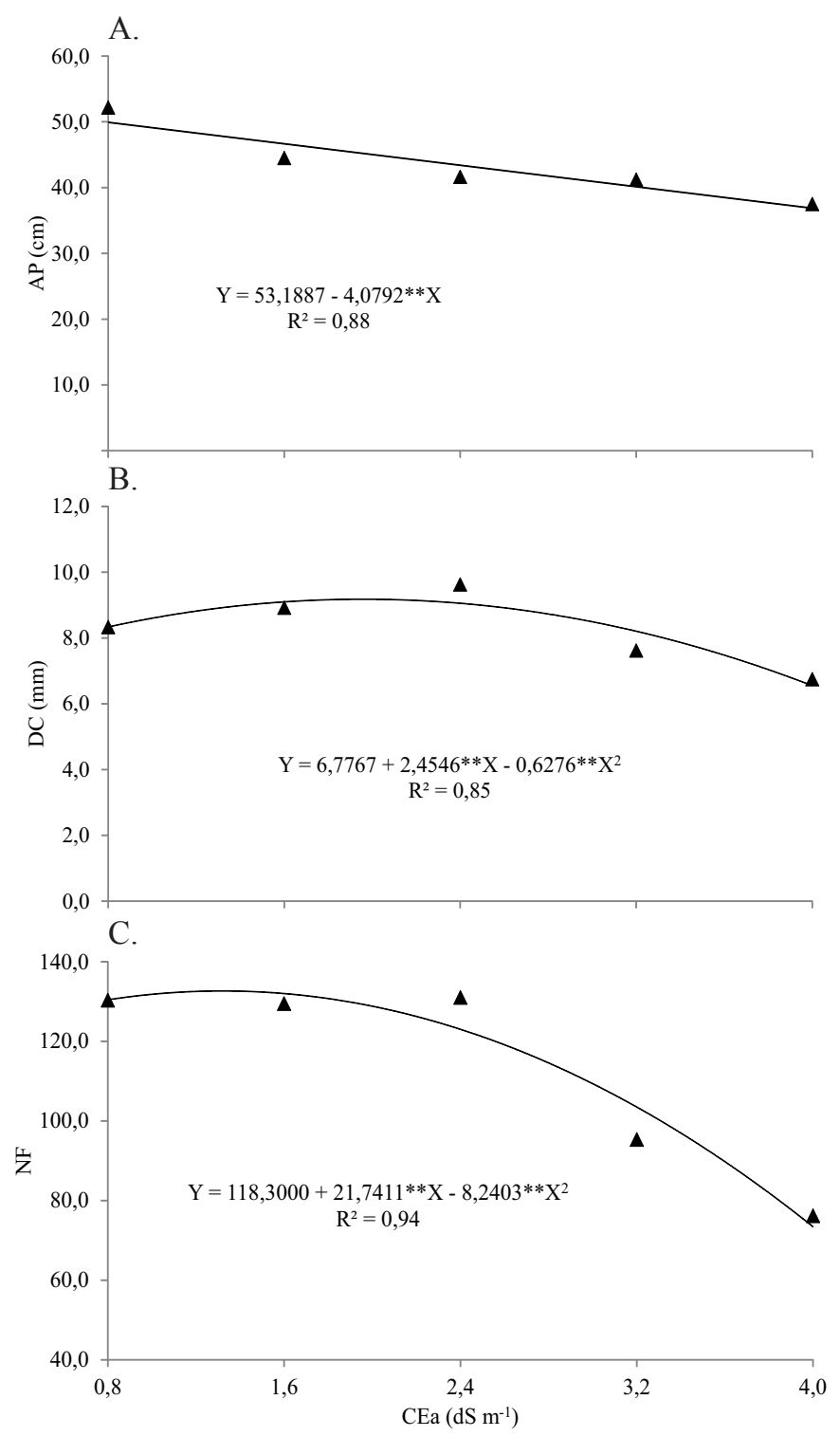

Figura 1. Altura de planta $(\mathrm{AP})(\mathrm{A})$, diâmetro de caule (DC) (B) e número de folhas (NF) (C) da espécie Crotalaria spectabilis em função da condutividade elétrica da água (CEa) de irrigação, aos 40 dias após aplicação dos tratamentos

Crotalaria juncea (Tabela 2) reforçando os resultados obtidos para altura, diâmetro e número de folhas para esta espécie, indicando ser a mesma tolerante a concentrações de níveis de sais da água de irrigação até $4,0 \mathrm{dS} \mathrm{m} \mathrm{m}^{-1}$ (Tabela 1 ).

Comportamento oposto foi obtido para a leguminosa Crotalaria spectabilis, a qual foi afetada pelos níveis de sais nas taxas de crescimento absoluto e relativo para altura de planta e diâmetro do caule, no período de 20-40 DAAT, confirmando os resultados obtidos para essas variáveis e número de folhas (Tabela 1). A altura ajustou-se ao modelo de regressão linear negativo com diminuição de $0,18 \mathrm{~cm} \mathrm{~d}^{-1}$ e $0,003 \mathrm{~cm} \mathrm{~cm}^{-1} \mathrm{~d}^{-1}$, nas taxas de crescimento absoluto e relativo, respectivamente (Figura 2A e 2C).

$\mathrm{O}$ diâmetro do caule ajustou-se ao modelo de regressão quadrático (Figura 2B e 2D), com efeito positivo da salinidade 
Tabela 2. Resumo de análise de variância para taxa de crescimento absoluto em altura (TCAa) e diâmetro (TCAd) e taxa de crescimento relativo em altura (TCRa) e diâmetro (TCRd) das espécies Crotalaria juncea e Crotalaria spectabilis irrigadas com águas de 0,8, 1,6, 2,4, 3,2 e 4,0 dS $\mathrm{m}^{-1}$ de condutividade elétrica, entre 0-20 e 20-40 dias após a aplicação dos tratamentos (DAAT)

\begin{tabular}{|c|c|c|c|c|c|c|c|c|c|}
\hline \multirow[b]{2}{*}{ Causa de variação } & \multirow[b]{2}{*}{ GL } & \multicolumn{2}{|c|}{ TCAa (DAAT) } & \multicolumn{2}{|c|}{ TCAd (DAAT) } & \multicolumn{2}{|c|}{ TCRa (DAAT) } & \multicolumn{2}{|c|}{ TCRd (DAAT) } \\
\hline & & \multicolumn{8}{|c|}{ Quadrados médios } \\
\hline & & \multicolumn{8}{|c|}{ Crotalaria juncea } \\
\hline Regressão linear & 1 & 0,40541 NS & 0,01544 NS & $0,00113^{\mathrm{NS}}$ & 0,00066 NS & 0,00001 NS & 0,00003 NS & 0,00000 NS & $0,00000 \mathrm{NS}$ \\
\hline Regressão quadrática & 1 & 0,08122 NS & 0,07140 NS & 0,00002 NS & 0,00008 NS & 0,00002 NS & 0,00005 NS & 0,00002 NS & 0,00000 NS \\
\hline Desvio regressão & 2 & $0,29961^{\mathrm{NS}}$ & 0,00780 NS & 0,00159 NS & 0,00104 NS & 0,00024 NS & $0,00001^{\mathrm{NS}}$ & $0,00000 \mathrm{NS}$ & $0,00007^{\mathrm{NS}}$ \\
\hline \multirow[t]{2}{*}{ CV $(\%)$} & & 21,26 & 11,02 & 26,32 & 2,15 & 16,14 & 0,51 & 0,73 & 24,65 \\
\hline & & \multicolumn{8}{|c|}{ Crotalaria spectabilis } \\
\hline Salinidade & 4 & 0,00309 NS & 0,38328 ** & 0,00103 NS & $0,01841^{\text {** }}$ & 0,00000 NS & $0,00014^{\text {** }}$ & 0,00016 NS & $0,00042^{* *}$ \\
\hline Regressão linear & 1 & 0,00508 NS & $1,31365^{* *}$ & 0,00101 NS & 0,03846 ** & 0,00000 NS & $0,00041^{\star *}$ & 0,00017 NS & $0,00103^{\text {** }}$ \\
\hline Regressão quadrática & 1 & 0,00000 NS & 0,11723 NS & 0,00023 NS & 0,01984 ** & 0,00000 NS & 0,00004 NS & 0,00000 NS & $0,00033^{* *}$ \\
\hline Desvio regressão & 2 & 0,00364 NS & 0,05113 NS & 0,00145 NS & $0,00767^{*}$ & 0,00000 NS & $0,00005^{\mathrm{NS}}$ & 0,00024 NS & 0,00016 ** \\
\hline
\end{tabular}

* $e{ }^{* \star}$ significativo a 0,05 e 0,01 de probabilidade, respectivamente; ${ }^{\text {NS }}$ não significativo

até o nível de 1,6 $\mathrm{dS} \mathrm{m}^{-1}$ para a taxa de crescimento absoluto e depreciativo deste nível em diante (Figura 2B).

A salinidade parece não influenciar a taxa de crescimento relativo do diâmetro até o nível de $1,6 \mathrm{dS} \mathrm{m}^{-1}$; porém se observa diminuição nessas taxas à medida que se elevou a concentração de sais na água a

A.

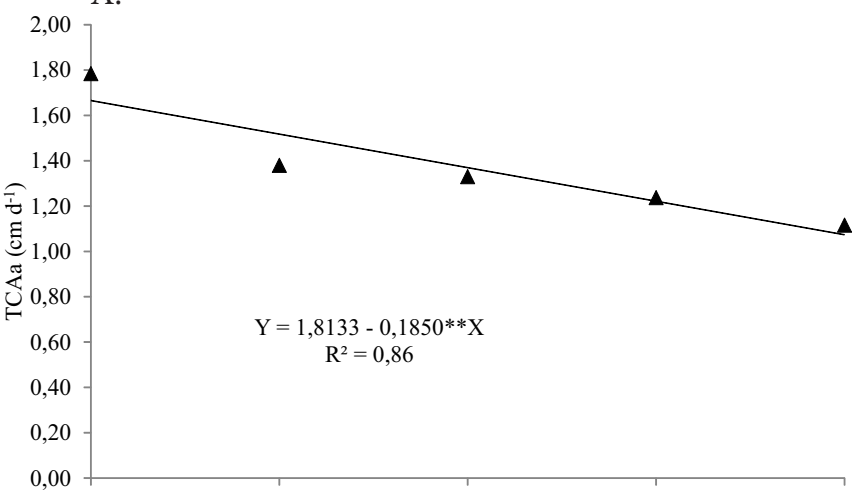

C.

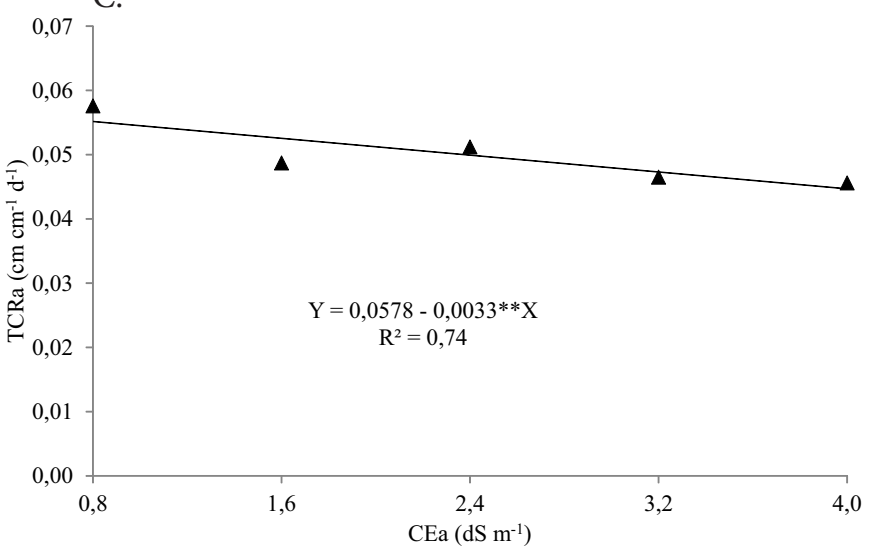

partir deste nível. É possível que esta redução no crescimento tenha sido decorrente do maior desvio de recursos energéticos para a osmorregulação dos sais nas plantas (Parida \& Das, 2005).

Távora et al. (2001) observaram acentuado decréscimo na TCR de plantas de goiabeira com o aumento da salinidade
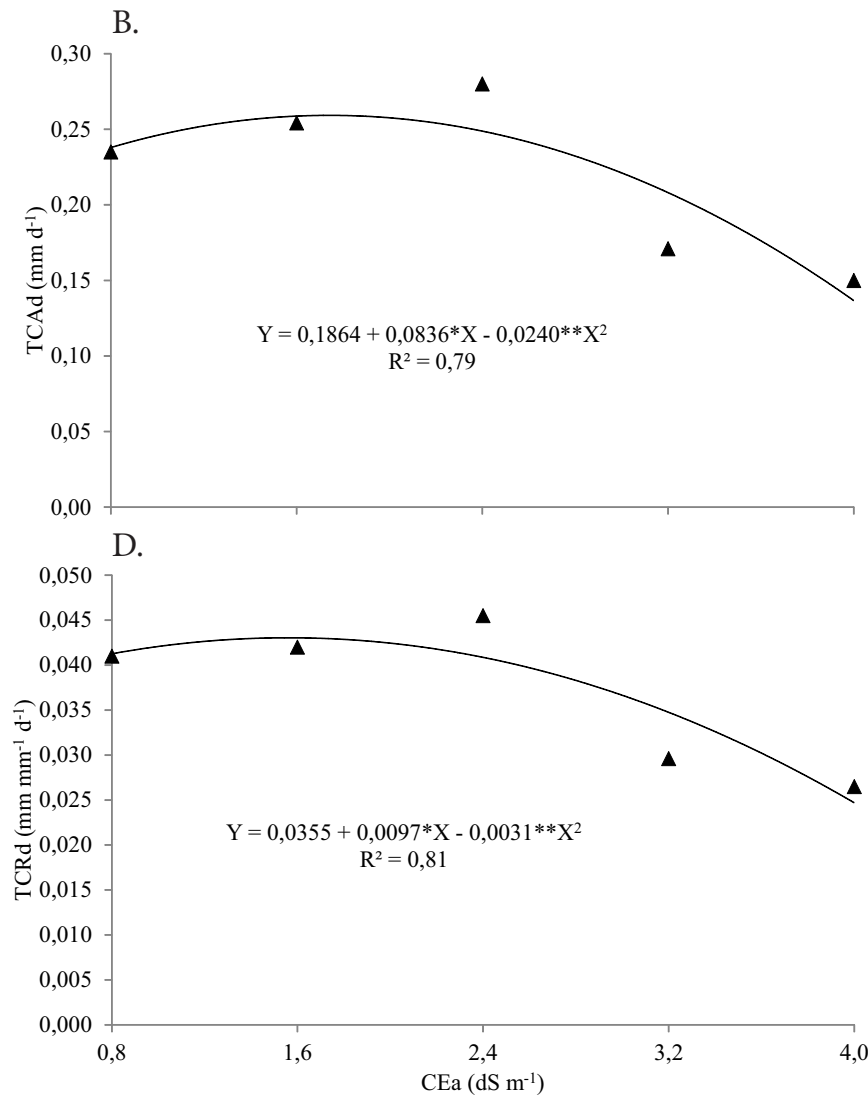

Figura 2. Taxa de crescimento absoluto em altura (TCAa) (A) e diâmetro (TCAd) (B) e taxa de crescimento relativo em altura (TCRa) (C) e diâmetro (TCRd) (D) da espécie Crotalaria spectabilis em função da condutividade elétrica da água (CEa) de irrigação, entre 20-40 dias após aplicação dos tratamentos 
verificando, na concentração máxima de $150 \mathrm{mmol} \mathrm{L}^{-1}$ de $\mathrm{NaCl}$, redução de cerca de 70\% no crescimento em relação ao controle.

As taxas de crescimento são indicativos importantes para inferir sobre a contribuição de processos fisiológicos e comportamento vegetal. Verifica-se redução nessas taxas quando ocorre excesso de sais nos tecidos das plantas com correlação negativa entre as concentrações salinas e o crescimento das plantas (Azevedo Neto \& Tabosa, 2000; Medeiros et al., 2007; Gurgel et al., 2010).

\section{Conclusões}

1. Os níveis de salinidade da água de irrigação não interferiram no crescimento da Crotalaria juncea até a condutividade elétrica de $4,0 \mathrm{dS} \mathrm{m}^{-1}$ no período de 40 dias.

2. A Crotalaria spectabilis não foi afetada pelos níveis de salinidade da água de irrigação, até os 20 dias da sua aplicação.

3. A salinidade da água de irrigação promoveu efeito negativo no crescimento da Crotalaria spectabilis, aos 40 dias, a partir da condutividade elétrica de $2,4 \mathrm{dS} \mathrm{m}^{-1}$.

4. A Crotalaria juncea tolerou os níveis de salinidade da água de irrigação por maior período de tempo que a Crotalaria spectabilis.

\section{Agradecimentos}

Os autores agradecem ao José Aparecido Donizeti, Diretor Comercial da Piraí Sementes, pelo fornecimento de sementes das leguminosas utilizadas nessa pesquisa.

\section{Literatura Citada}

Alcântara, F. A.; Furtini Neto, A. E.; De Paula, M. B.; Mesquita, H. A.; Muniz, J. A. Adubação verde na recuperação da fertilidade de um Latossolo vermelho-escuro degradado. Pesquisa Agropecuária Brasileira, v.35, p.277-288, 2000.

Amorim, J. R. A.; Fernandes, P. D.; Gheyi, H. R.; Azevedo, N. C. Efeito da salinidade e modo de aplicação da água de irrigação no crescimento e produção de alho. Pesquisa Agropecuária Brasileira, v.37, p.167-176, 2002.

Azevedo Neto, A. D.; Tabosa, J. N. Estresse salino em plântulas de milho: parte I - análise do crescimento. Revista Brasileira de Engenharia Agrícola e Ambiental, v.4, p.159-164, 2000.

Benincasa, M. M. P. Análise de crescimento de plantas. Jaboticabal: FUNEP, 2003. 41p.

Bustingorri, C.; Lavado, R. S. Soybean growth under stable versus peak salinity. Scientia Agricola, v.68, p.102-108, 2011.

Carneiro, M. A. C.; Cordeiro, M. A. S (3); Assis, P. C. R.; Moraes, E. S.; Pereira, H. S.; Paulino, H. B.; Souza, E. D. Produção de fitomassa de diferentes espécies de cobertura e suas alterações na atividade microbiana de solo de cerrado. Bragantia, v.67, p.455-462, 2008.
Cunha, E. de Q.; Stone, L. F.; Ferreira, E. P. B.; Didonet, A. D.; Moreira, J. A. A.; Leandro, W. M. Sistemas de preparo do solo e culturas de cobertura na produção orgânica de feijão e milho: Parte II - Atributos biológicos do solo. Revista Brasileira de Ciência do Solo, v.35, p.603-611, 2011.

EMBRAPA - Empresa Brasileira de Pesquisa Agropecuária. Sistema Brasileiro de Classificação do Solo. Brasília: Embrapa, 2006. 306p.

Ferreira, D. F. SISVAR: A computer statistical analysis system. Ciência e Agrotecnologia, v.35, p.1039-1042, 2011.

Fontanétti, A.; Carvalho, G. J.; Gomes, L. A. A.; Almeida, K.; Moraes, S. R. G.; Teixeira, C. M. Adubação verde na produção orgânica de alface americana e repolho. Horticultura Brasileira, v.24, p.146-150, 2006.

Gurgel, M. T.; Uyeda, C. A.; Gheyi, H. R.; Oliveira, F. H. T.; Fernandes, P. D.; Silva, F. V. Crescimento de meloeiro sob estresse salino e doses de potássio. Revista Brasileira de Engenharia agrícola e Ambiental, v.14, p.3-10, 2010.

Kudo, N.; Sudino, T.; Oka, M.; Fujiyama, H. Sodium toleran ce of plants in relation to ionic balance and the absorption ability of microelementos. Soil Science and Plant Nutrition, v.56, p.225-233, 2010.

Mahajan, S.; Tuteja, N. Cold, salinity and drought stresses: An overview. Archives of Biochemistry and Biophysics, v.444, p.139-158, 2005.

Medeiros, J. F.; Silva, M. C. C.; Sarmento, D. H. A.; Barros, A. D. Crescimento do meloeiro cultivado sob diferentes níveis de salinidade, com e sem cobertura do solo. Revista Brasileira de Engenharia Agrícola e Ambiental, v.11, p.248-255, 2007.

Mendonça, A.V. R.; Carneiro, J. G. A.; Barroso, D. G.; Santiago, A. R.; Rodrigues, L. A.; Freitas, T. A. S. Características biométricas de mudas de Eucalyptus sp sob estresse salino. Árvore, v.31, p.365-372, 2007.

Nery, A. R.; Rodrigues, L. N.; Silva, M. B. R. da; Fernandes, P.D.; Chaves, L. H. G.; Dantas Neto, J.; Gheyi, H. R. Crescimento do pinhão-manso irrigado com águas salinas em ambiente protegido. Revista Brasileira de Engenharia Agrícola e Ambiental, v.13, p.551-558, 2009.

Neves, A. L. R.; Lacerda, C. F.; Guimarães, F. V. A.; Hernandez, F. F. F.; Silva, F. B.; Priscol, J. T.; Gheyi, H. R. Acumulação de biomassa e extração de nutrientes por plantas de feijãode-corda irrigadas com água salina em diferentes estádios de desenvolvimento. Ciência Rural, v.39, p.758-765, 2009.

Nunes, A. S.; Lourenção, A. L. S.; Pezarico, C. R.; Scalon, S. P. Q.; Gonçalves, M. C. Fontes e níveis de salinidade na germinação de sementes de Crotalaria juncea L.. Ciência e Agrotecnologia, v.33, p.753-757, 2009.

Oliveira, F. A.; Medeiros, J. F.; Oliveira, M. K. T.; Lima, C. J. G. de S.; Almeida Júnior, A. B.; Amâncio, M. G. Desenvolvimento inicial do milho-pipoca irrigado com água de diferentes níveis de salinidade. Revista Brasileira de Ciências Agrárias, v.4, p.149-155, 2009.

Parida, A. K.; Das, A. B. Salt tolerance and salinity effects on plants: A review. Ecotoxicology and Environmental Safety, v.60, p.324-349, 2005.

Richards, L. A. (ed.). Diagnosis and improvement of saline and alkali soils. Washington: U.S. Salinity Laboratory, 1954. 160p. USDA. Agriculture Handbook, 60 
Santos, R. S.; Dantas, D. C.; Nogueira, F. P.; Dias, N. da S.; Ferreira Neto, M.; Gurgel, M. T. Utilização de águas salobras no cultivo hidropônico da alface. Irriga, v.15, p.111-118, 2010.

Silva, F. E. O.; Maracajá, P. B.; Medeiros, J. F. de; Oliveira, F. A.; Oliveira, M. K. T. Desenvolvimento vegetativo do feijão caupi irrigado com água salina em casa de vegetação. Revista Caatinga, v.22, p.156-159, 2009.

Silva, S. M. S.; Alves, A. N.; Gheyi, H. R.; Beltrão, N. E. de M.; Severino, L. S.; Soares, F. A. L. Desenvolvimento e produção de duas cultivares de mamoneira sob estresse salino. Revista Brasileira de Engenharia Agrícola e Ambiental, v.12, p.335342, 2008.
Távora, F. J. A. F.; Ferreira, R. G.; Hernandez, F. F. F. Crescimento e relações hídricas em plantas de goiabeira submetidas a estresse salino com $\mathrm{NaCl}$. Revista Brasileira de Fruticultura, v.23, p.441-446, 2001.

Vargas, T. O.; Diniz, E. R.; Santos, R. H. S.; Lima, C. T. A.; Urquiaga, S.; Cecon, P. R. Influência da biomassa de leguminosas sobre a produção de repolho em dois cultivos consecutivos. Horticultura Brasileira, v.29, p.562-568, 2011.

Zhu, J. K. Plant salt tolerance. Trends in plant science, v.6, p.66-71, 2001.

Zhu, J.; Zhilong, B.; Li, Y. Physiological and growth responses of two different salt-sensitive cucumber cultivars to $\mathrm{NaCl}$ stress. Soil Science Plant Nutrition, v.54, p.400-407, 2008. 